\title{
An ultra-sensitive localised surface plasmon resonance fibre device for environmental sensing based upon a structured bi-metal coating
}

\author{
T. Allsop, R. Neal ${ }^{+}$, C. Mou, K. Kalli*, D.J. Webb \\ Authors' Affiliations \\ Aston Institute of Photonic Technologies, Aston University, Aston Triangle, Birmingham, B47ET, \\ UK. \\ ${ }^{+}$Faculty of Technology, University of Plymouth, Plymouth, PL4 8AA, U.K. \\ *Cyprus University of Technology, Limassol 3036, Cyprus.
}

\begin{abstract}
We demonstrate a bi-metal coating (platinum and gold or silver) localised surface plasmon resonance fibre device that produces an index spectral sensitivity of over $11,000 \mathrm{~nm} / \mathrm{RIU}$, yielding an index resolution of $5 \times 10^{-6}$ in the aqueous index regime, consisting of a structured multi-layered thin film on D-shaped fibre
\end{abstract}

\section{Introduction}

Fibre optic sensors based upon grating structures - including long period gratings (LPGs), fibre Bragg gratings (FBGs) and tilted fibre Bragg gratings (TFBGs) - and surface plasmon resonances (SPR) (localised, damped and conventional) may all be used to detect changes in the refractive index of various substances with potential applications in chemistry, biochemistry and biology ${ }^{1-4}$. This sensitivity is characterised by changes in the spectral wavelength and optical strength of features in the transmission spectrum of the sensors. In SPR-based sensors these features are particularly sensitive to the polarisation of the illuminating light and have been used to detect index changes in biochemical/chemical reactions ${ }^{5}$. Surface plasmons can be divided into three types; the long range surface plasmon (LRSP) ${ }^{6}$, the short range surface plasmon (SRSP) $)^{6}$ and the localised surface Plasmon (LSP) ${ }^{7}$, all types being generated at a metal/dielectric interface:. Recent focus on the generation of LSPs results from the production of various surface topologies and the use of novel materials (structured thin films), which in turn governs the optical and physical characteristics of the plasmons being generated. The LRSP and SRSP obey the following dispersion relation for two homogeneous semi-infinite media:

$$
\beta=k \sqrt{\left(\frac{\varepsilon_{m} \cdot n_{s}{ }^{2}}{\varepsilon_{m}+n_{s}{ }^{2}}\right)}
$$

where $k$ is the free space wave number, $\varepsilon_{m}$ is the dielectric constant of the metal $\left(\varepsilon_{m}=\varepsilon_{m r}+i \varepsilon_{m i}\right)$ and $n_{s}$ is the refractive index of the dielectric sample to be tested. For LSP a modified dispersion relation can be evaluated based on a corrugated nano structure, with the presence of apertures in the supporting surface plasmon material. This array of apertures $^{8}$ has a momentum component of light parallel to the surface, $k_{x}=\frac{2 \pi}{p} \cdot \sqrt{i^{2}+j^{2}}$, where $P$ is a lattice constant (the distance between the apertures), $i$ and $j$ are nonzero integer numbers representing the scattering orders from the two dimensional aperture arrays. This is the resonant condition for the localised surface plasmons that needs to be satisfied for a lattice structure ${ }^{7}$.

The complex spectral behaviour of LSPs is determined by many physical dependencies, for example on the size, topology and geometries of the plasmon-supporting nanostructures. Examples of the effects of surface topology on the spectral behaviour of plasmons can readily be found in the literature ${ }^{4,8}$. The optical properties and characteristics of structured thin film can be comprehensively tuned by nano-engineering the structure parameters, such as using periodically arranged nanopores or microporous and nanoporous metal structures ${ }^{8,9}$.

We report on a novel localised SPR fibre device based upon an ultra-violet inscribed surface relief grating-type structure in a multi-layered thin film deposited on the flat side of a lapped D-shaped fibre, which exhibits the highest spectral sensitivity to refractive index in the aqueous index regime for a fibre optic sensor.

\section{Fabrication and Characterisation}

The SPR fibre devices are constructed in three stages ${ }^{9}$. Firstly, a standard SMF fibre is mechanically lapped down to $6 \mu \mathrm{m}$ from the core-cladding interface. Secondly, using the RF sputtering technique, a series of coatings is deposited upon the flat of the lapped fibre with materials and average thicknesses of: $\mathrm{Ge}$ of $48 \mathrm{~nm}, \mathrm{SiO}_{2}$ of $48 \mathrm{~nm}$, followed by a series of ultra-thin coatings consisting of alternating layers of platinum and gold or silver with individual thicknesses of 3nm per 
layer with a total thickness of 36nm. Thirdly, the coated lapped fibre is exposed to a diffracted pattern of UV light from a uniform phase mask (through laser beam scanning and multi-exposures), see figure 1 . This produces a surface relief structure which has approximate and predominant periods of $\sim 0.6 \mu \mathrm{m}$ and $\sim 1 \mu \mathrm{m}$, with a corrugation thickness of the order of the bi-metal layer thus producing rods of bimetal on the flat of the D-shaped fibre; the mechanism and details of the processes creating these structures have been reported elsewhere ${ }^{9}$. The approximate dimensions of the nanorods are length of $12 \mu \mathrm{m}$ and mean diameter 350nm.

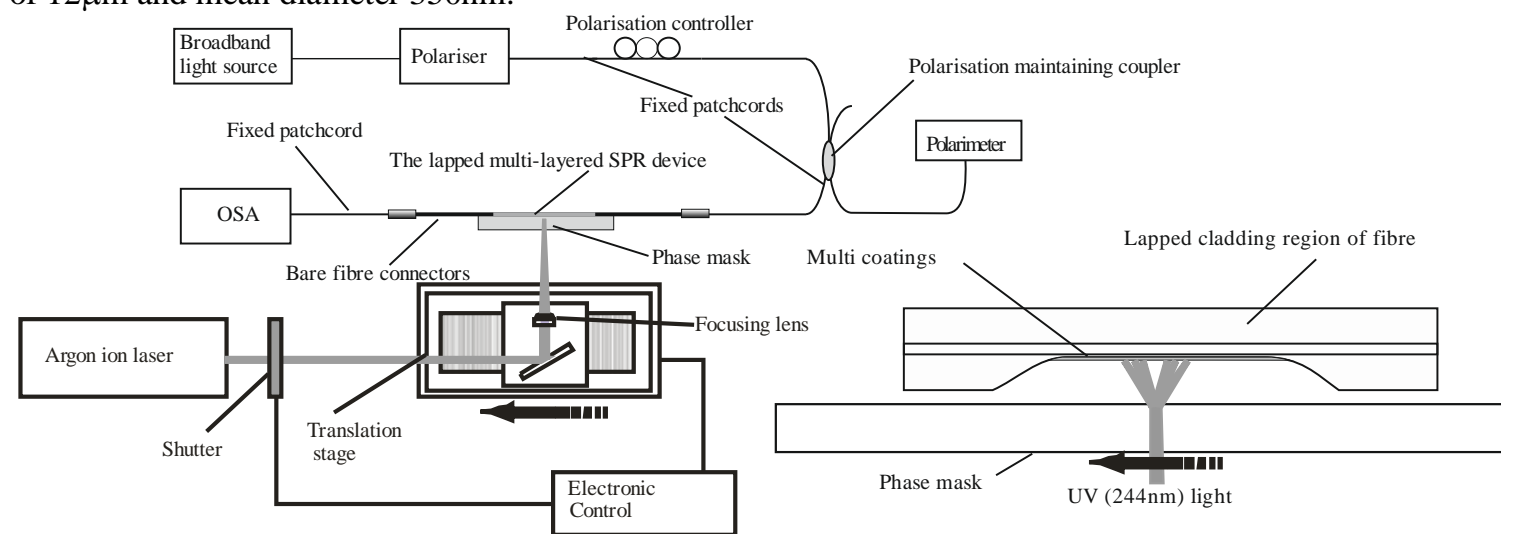

Figure 1. Scheme used for the fabrication of the nano-scale structured coating and the equipment used to characterise the devices
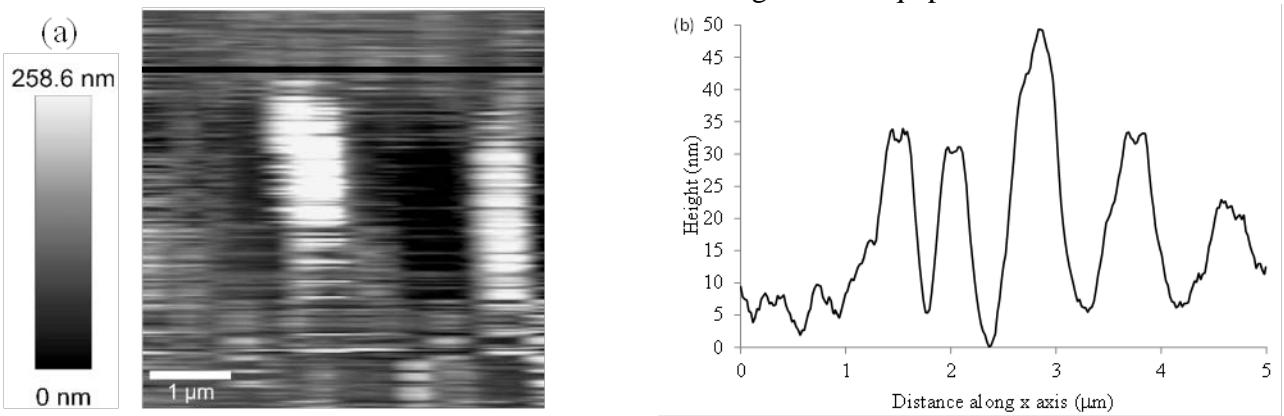

Figure 2. (a) AFM image of the surface nano-scaled structured coating on the lapped region of the optical fibre, (b) the surface line profile depicted by the black line shown in (a).

\section{Sensing Spectral Characteristics}

The spectral sensitivity to refractive index and polarisation of the transmission spectral features of the LSP fibre devices are investigated before, during and after formation of this structure. Figure 1 shows the experimental set-up used for polarisation characterisation, both during fabrication and when the fibre devices are submerged into various liquids with different refractive indices; Cargille refractive index matching solutions. Light from a broadband light source was passed through a polariser and a polarisation controller before illuminating the sample, with the transmission spectrum being monitored using an optical spectrum analyser.
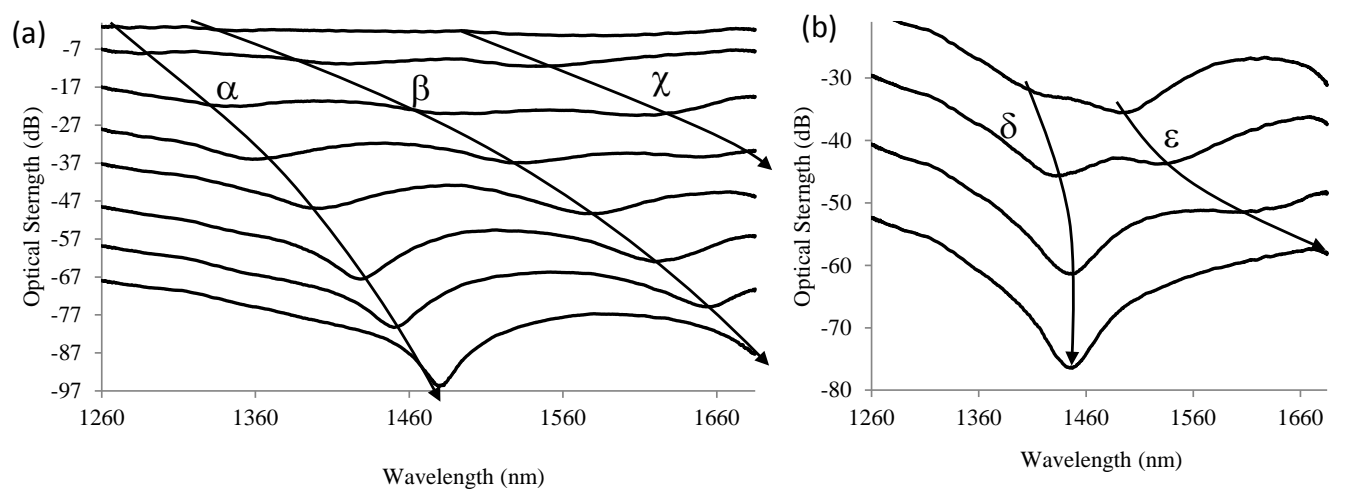

Figure 3 Transmission spectra of $\mathrm{GelSiO}_{2} \backslash \mathrm{PtAu}$ as a function of refractive index of surrounding medium, (a) index variation from 1 to 1.36 and (b) index variation from 1.365 to 1.395 . 
Table 1 index sensitivities for various index ranges of PtAu Device prior to UV processing

\begin{tabular}{c|c|c|c} 
Plasmon Resonance & index & Wavelength Sensitivity nm/RIU & Intensity Sensitivity dB/RIU \\
\hline \hline$\alpha$ & 1.30 to 1.34 & 3020 & 259 \\
$\beta$ & 1.30 to 1.34 & 4170 & 248 \\
$\chi$ & 1.30 to 1.34 & 3930 & 132 \\
\hline$\alpha$ & 1.34 to 1.36 & 5050 & 816 \\
$\beta$ & 1.34 to 1.36 & 7060 & 450 \\
$\chi$ & 1.34 to 1.36 & out of measurement range & out of measurement range \\
\hline$\delta$ & 1.365 to 1.395 & 1300 & 452 \\
$\varepsilon$ & 1.365 to 1.396 & 9220 & -303
\end{tabular}

Prior to UV processing there are three plasmon resonances occurring over the observable wavelength range; $1260 \mathrm{~nm}$ to $1690 \mathrm{~nm}$, see figure 3; their index sensitivities are shown in Table 1 with the $\varepsilon$ resonance having greatest sensitivity and yielding an index resolution of $\sim 6 \times 10^{-6}$ in the aqueous index regime (assuming a $0.05 \mathrm{~nm}$ wavelength resolution). The polarisation behaviour of the three resonances was also studied, for which a zero set-point was chosen for the strongest optical feature in transmission using an index solution of 1.360, and occurring at 1448nm (labelled resonance $\beta$ ). Rotation of the azithumal polarisation changed the resonant wavelengths and the associated optical strengths,shown in figure 4. It is expected that for different wavelength resonances the comparative polarisation dependence would be different along with the wavelength shifts; this is shown in figure 4 for different materials. Moreover these resonances are probably generated by conventional damped surface plasmons, this is suggested by the fact that the resonances have reasonably large optical strength over a large range of azimuthal polarisation of the illuminating light.
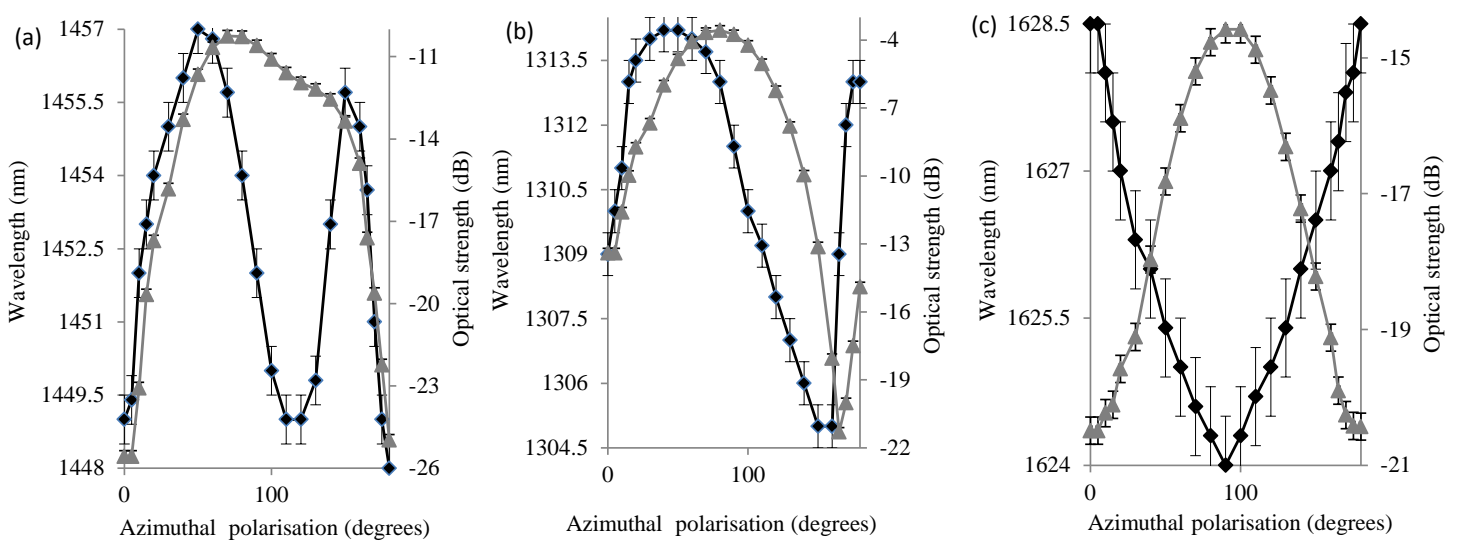

Figure 4. The variation in central wavelength and coupling efficiency of the SPRs as a function of change of polarisation azimuth at the polarisation controller. The angle is measured from the point of maximum coupling strength of the main transmission feature in an index solution of 1.360 . The device is $\mathrm{Au} / \mathrm{Pt}-\mathrm{SiO}_{2}-\mathrm{Ge}$ coated with the main three surface plasmon resonances investigated. The maximum transmission feature is at $1448 \mathrm{~nm}$ for resonance $\beta$ (a), resonance $\alpha$ (b) and resonance $\chi(\mathrm{c}),(\boldsymbol{\nabla})$ is the wavelength shift ( $\Delta$ ) is the optical strength variation

After the UV processing to produce the nano-scaled surface structure, the refractive index sensitivity and polarisation dependence was investigated again, figures 5 , and the index response summarised in Table 2.

Table 2 index sensitivities for various index ranges of the Pt/Au Device after UV processing

\begin{tabular}{c|c|c|c} 
Plasmon Resonance & index & Wavelength Sensitivity nm/RIU & Intensity Sensitivity dB/RIU \\
\hline \hline$\phi$ & 1.33 to 1.36 & 3430 & 331 \\
$\gamma$ & 1.30 to 1.35 & 3830 & 248 \\
$\eta$ & 1.30 to 1.33 & 3470 & 124 \\
\hline$\phi$ & 1.36 to 1.395 & 11250 & -314 \\
$\gamma$ & 1.36 to 1.395 & 6250 & 450 \\
$\eta$ & 1.33 to 1.35 & 6980 & 130 \\
\hline
\end{tabular}

There are very large spectral sensitivities with regards to index change, see Table 2 . In particular the $\phi$ resonance shows a spectral index sensitivity of $11250 \mathrm{~nm} / \mathrm{RIU}$ in the higher aqueous range that leads to index resolution of $\sim 4 \times 10^{-6}$. Furthermore, the resonances appear to have a higher sensitivity to the polarisation state of the illuminating light yielding a maximum polarisation sensitivity of $0.502 \mathrm{~dB}$ /degree, compared to $0.398 \mathrm{~dB} /$ degree before $\mathrm{UV}$ processing. This is 
expected and the authors have reported this elsewhere ${ }^{9,10}$ and yields another physical property that can be exploited as a sensing mechanism ${ }^{11}$.
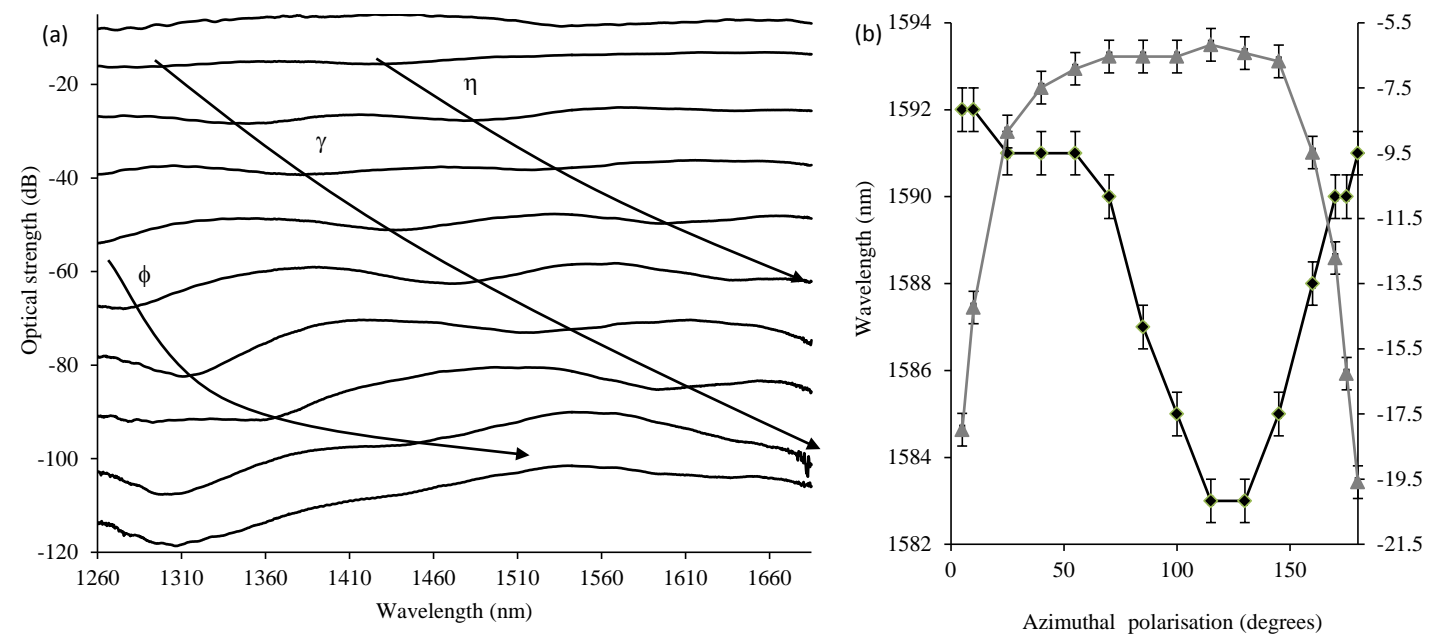

Figure 5 (a) Transmission spectra of $\mathrm{GelSiO}_{2} \mathrm{LtAu}$ as a function of the refractive index of the surrounding medium after UV processing with index variation from 1.3 to 1.395 , (b) the variation in central wavelength and coupling efficiency of the SPRs as a function of change of polarisation azimuth at the polarization controller. The angle is measured from the point of maximum coupling strength of main transmission feature in an index solution of 1.360 . The main transmission feature at $1580 \mathrm{~nm}$, the polarisation dependence of resonance $\eta(a),(\bullet)$ is the wavelength shift $(\boldsymbol{\Delta})$ is the optical strength variation.

The bi-metal coating (platinum and gold) localised surface plasmon resonance fibre device yields a bulk index spectral sensitivity of $11250 \mathrm{~nm} / \mathrm{RIU}$. This is one the highest index sensitivities in the aqueous index regime, compared to other various fibre sensing techniques ${ }^{10,12}$. Also this device has a greater sensitivity than a single metal overlay nanostructured coated fibre optic sensor, which demonstrated 4000 to $9800 \mathrm{~nm} / \mathrm{RIU}^{13}$. A main advantage of this approach is ease of coupling and interrogation over the planar devices; at the same time itapproaches the performance of some of the newly developed structured materials for bio-sensing ${ }^{14}$.

\section{Acknowledgments}

The Authors would like thank WITec GmbH in Ulm, Germany and in particular Dr Thomas Dieing for the use of their equipment resulting in data shown in figure 2 .

\section{References}

[1] 1 S. Vasilev et al, "Long-period refractive index fibre gratings: properties, applications and fabrication techniques", Proc. SPIE 4083, pp.212-223,2000.

[2] K. Schroeder et al, “A fibre Bragg grating refractometer”, Meas. Sci. Technol. 12, pp.757-764, 2001.

[3] M. Piliarik et al, "Surface plasmon resonance sensor based on a single-mode polarisation-maintaining optical fiber", Sensors and Actuators B, Vol. 90, pp.236-242, 2004

[4] T. Allsop, et al, "The exploitation of multilayer coatings for infra-red surface plasmon resonance fibre sensors", Appl. Optics 48(1), 276-286 (2009).

[5] J. Homola, "Surface plasmon resonance sensors: review", Sensors and Actuators B, Vol. 54, pp.3-15,1999.

[6] "'Surface Plasmons on smooth and Rough Surfaces and on Gratings", H. Raether, eds. (Academic, New York, 1997).

[7] E. Hutter, et al, "Exploitation of localized surface plasmon resonance," Advanced Materials 16(19), 1685-1706 (2004).

[8] D. C. Skigin et al, "Study of resonant modes of a periodic metallic array near a dielectric interface: evanescent-to-propagating coupling via surface plasmon excitation,” J. Opt. A: Pure Appl. Opt. 8, 259-267 (2006).

[9] T. Allsop, et al, "Formation and characterisation of ultra-sensitive surface plasmon resonance sensor based upon a nano-scale corrugated multi-layered coated D-shaped optical fibre,” Jn. Quantum Electron. 48(3), 394 - 405, (2012).

[10] S. Roh et al, "Overview of the Characteristics of Micro- and Nano-Structured Surface Plasmon Resonance Sensors", Sensors, 11, pp.1565-1588, (2011).

[11] M. Piliarik, et al, "Surface plasmon resonance sensor based on a single-mode polarisation-maintaining optical fiber. Sensors and Actuators B, Vol. 90, pp.236-242, (2004)

[12] J. Chen, et al, "High-sensitivity fiber refractive index sensor formed by sandwiching a thinned taper between two abrupt tapers", Proc. SPIE 9044, International Conference on Optical Instruments and Technology, 90440D, (2013).

[13] B. Lee, "Waveguide-based surface plasmon resonance sensor design”, Proc. SPIE, 7420, 74200C, (2009).

[14] A.V. Kabashn, et al, "Plasmonic nanorod metamaterials for biosensing”, Nat. Material, 8, 867-871, (2009) 